\title{
Subverting lysosomal function in Trypanosoma brucei
}

\author{
Sam Alsford* \\ London School of Hygiene \& Tropical Medicine, Keppel Street, London WC1E 7HT, UK. \\ * Corresponding Author: Sam Alsford, Tel: +44 (0)20 7927 2352; E-mail: sam.alsford@lshtm.ac.uk
}

\begin{abstract}
In this issue of Microbial Cell, Koh and colleagues present data highlighting the utility of the lysosomotropic compound L-leucyl-L-leucyl methyl ester (LeuLeu-OMe) as an anti-Trypanosoma brucei agent, adding to the range of compounds that either directly target lysosomal enzymes or that can be used to subvert the function of the lysosome for parasite destruction.
\end{abstract}

Trypanosoma brucei causes devastating diseases in humans and cattle [1]. Human African trypanosomiasis (HAT), caused by $T . b$. gambiense and $T$. $b$. rhodesiense, and the livestock disease Nagana, caused by $T$. brucei and related parasites, are endemic to sub-Saharan Africa, with approximately 60 million people living in at risk areas [2]. Due to sustained control efforts over the last two decades, HAT cases have dropped from an estimated peak of 300-500 thousand per year in the 1990 s, to less than 10,000 reported cases per year since 2009 [3]. Given this success, the World Health Organisation has stated that HAT should be eliminated as a public health problem by 2020 [4]. However, $T$. brucei and the related zoonotic African trypanosomes, $T$. congolense and $T$. vivax, still present a significant constraint on agricultural development in the region. Meeting the HAT elimination goal in an environment of widespread zoonotic trypanosomiasis will likely require the development of new chemotherapeutic interventions for both HAT and Nagana. The current arsenal of anti-HAT drugs is limited by disease stage and sub-species specificity, as well as toxicity and the need for complex administration protocols [5]. In addition, resistance to the anti-HAT drugs, melarsoprol and pentamidine, has been extensively characterised in clinical isolates [6], and treatment failures with the anti-animal trypanosomiasis drugs, diminazene aceturate and isometamidium chloride are also seen [7].

A small number of new compounds are currently under clinical development for the treatment of HAT, including orally available oxaboroles [8] and fexinidazole [9]. Work is also ongoing to identify specific trypanosomal drug targets, inspired by the array of unique features present in this evolutionarily divergent eukaryote, which represent attractive targets for chemotherapeutic intervention [10]. Given this, it is perhaps surprising that the lysosome, an organelle found in all eukaryotes, has emerged as a promising drug target. Cathepsin-L, an essential T. brucei lysosomal cysteine protease [11] and its homologue in the related trypanosomatid $T$. cruzi, the causative agent of Chagas disease, are the subject of intense research [12], with the aim of developing selective inhibitors. Several other anti-parasitic agents either depend on proper lysosomal function for their efficacy, such as the anti-HAT drug suramin [13], or impact its function directly, such as the anti-malarial chloroquine [14]. In this edition of Microbial Cell, Koh and colleagues highlight the anti-trypanosomal potential of other lysosomotropic compounds, demonstrating the selectivity of LeuLeu-OMe for bloodstream form T. brucei and its likely mode of killing [15]. LeuLeu-OMe has previously been shown to cause lysosomal membrane permeabilisation (LMP) in Plasmodium falciparum, though only at millimolar concentrations [14]. With an $\mathrm{EC}_{50}$ of $16 \mu \mathrm{M}$, its potency against $T$. brucei is considerably higher [15], although this will need to be further improved, if LeuLeu-OMe and related compounds are to have a future role in HAT chemotherapy.

LeuLeu-OMe exhibits some toxicity against mammalian cells, with selectivity for cytotoxic lymphocytes [16], but even these cells are less sensitive to this compound than $T$. brucei [15]. Treatment of HeLa cells with $>300 \mu \mathrm{M}$ LeuLeuOMe results in LMP and the release of cathepsins into the cytosol, which cleave the key apoptosis regulator, Bid, leading to caspase activation and apoptosis [17]. The phenotypes seen in $P$. falciparum following LMP are also reminiscent of apoptosis, and include mitochondrial membrane depolarisation and DNA fragmentation [14]. Apoptosis-like features have also been reported in $T$. brucei and other protozoa [18] however the absence of the key regulators and the caspase executioners, and the lack of convincing evidence for regulation of the process have led others to conclude that the observed cell death should be described as incidental or necrotic [19]. Indeed, although cathepsin release following LMP can lead to apoptosis in metazoa [17], the data presented by Koh and colleagues indicate that LeuLeu-OMe mediated LMP in T. brucei causes necrotic cell death, without any discernible markers of apoptosis [15]. One or both of the parasite's cathepsins ( $B$ and $L$ ) 
appears to be at least partly responsible for the observed necrosis, as incubation of LeuLeu-OMe treated cells with a broad range cathepsin inhibitor significantly reduces cell death [15].

The lysosome has long been recognised as a viable target for chemotherapeutic intervention that subverts its function for parasite destruction. Indeed, humans are resistant to infection by the non-human infectious African trypanosomes, such as $T$. congolense and $T$. b. brucei, due to serum factors that target the parasite's lysosome. Human serum contains two trypanolytic complexes, which rapidly lyse non-human infectious African trypanosomes: trypanolytic factor (TLF) 1 is a component of high density lipoprotein, while TLF2 is composed primarily of IgM [20]. The lytic component of both complexes is apolipoproteinL1, which forms pores in the lysosomal membrane following a conformational change at low $\mathrm{pH}$, allowing the uncontrolled entry of chloride ions into the lysosome and its subsequent osmotic swelling leading to parasite lysis [21]. The lysosome and the endocytic system that feeds into it are also attractive drug targets by virtue of their fundamental role in surface coat recycling and antigenic variation, the process whereby $T$. brucei changes its variant surface glycoprotein coat, enabling immune evasion [22] and the rapid removal of bound antibody [23]. It may be these features, the very high endocytic rate and a necessarily highly active lysosome, that render bloodstream form $T$. brucei more sensitive than human cells to LeuLeu-OMe.

\section{REFERENCES}

1. Brun R, Blum J, Chappuis F, Burri C (2010). Human African trypanosomiasis. Lancet 375(9709): 148-159.

2. Simarro PP, Cecchi G, Franco JR, Paone M, Diarra A, Ruiz-Postigo JA, Fevre EM, Mattioli RC, Jannin JG (2012). Estimating and mapping the population at risk of sleeping sickness. PLoS Neglected Tropical Diseases 6(10): e1859.

3. Simarro PP, Cecchi G, Franco JR, Paone M, Diarra A, Priotto G Mattioli RC, Jannin JG (2015). Monitoring the Progress towards the Elimination of Gambiense Human African Trypanosomiasis. PLoS Neglected Tropical Diseases 9(6): e0003785.

4. WHO (2012). Accelerating Work to Overcome the Global Impact of Neglected Tropical Diseases. A roadmap for implementation. Executive Summary. World Health Organisation, Geneva.

5. Barrett MP, Croft SL (2012). Management of trypanosomiasis and leishmaniasis. British Medical Bulletin 104: 175-196.

6. Graf FE, Baker N, Munday JC, de Koning HP, Horn D, Maser P (2015). Chimerization at the AQP2-AQP3 locus is the genetic basis of melarsoprol-pentamidine cross-resistance in clinical Trypanosoma brucei gambiense isolates. International Journal for Parasitology Drugs and Drug Resistance 5(2): 65-68.

7. Mungube EO, Vitouley HS, Allegye-Cudjoe E, Diall O, Boucoum Z, Diarra B, Sanogo Y, Randolph T, Bauer B, Zessin KH, Clausen PH (2012). Detection of multiple drug-resistant Trypanosoma congolense populations in village cattle of south-east Mali. Parasites and Vectors 5: 155.
The importance of the endosomal-lysosomal system to $T$. brucei survival in the mammalian host, both as a route for nutrient uptake and for surface coat maintenance, underpins its successful targeting by both the innate immune system and chemotherapy [24]. Any new compounds that target this area are an attractive proposition. Whether, LeuLeu-OMe represents a significant step forward will depend on the ease with which more potent and highly selective variants can be developed.

\section{ACKNOWLEDGMENTS}

Work in my laboratory is supported by Medical Research Council/Department for International Development project grant (MR/K011987/1).

\section{CONFLICT OF INTEREST}

I have no conflicts of interest.

\section{COPYRIGHT}

(C) 2015 Alsford. This is an open-access article released under the terms of the Creative Commons Attribution (CC BY) license, which allows the unrestricted use, distribution, and reproduction in any medium, provided the original author and source are acknowledged.

Please cite this article as: Sam Alsford (2015). Subverting lysosomal function in Trypanosoma brucei. Microbial Cell 2(8): 259-261. doi: $10.15698 / \mathrm{mic} 2015.08 .222$

8. Jacobs RT, Nare B, Wring SA, Orr MD, Chen D, Sligar JM, Jenks MX, Noe RA, Bowling TS, Mercer LT, Rewerts C, Gaukel E, Owens J, Parham R, Randolph R, Beaudet B, Bacchi CJ, Yarlett N, Plattner JJ, Freund Y, Ding C, Akama T, Zhang YK, Brun R, Kaiser M, Scandale I, Don R (2011). SCYX-7158, an orally-active benzoxaborole for the treatment of stage 2 human African trypanosomiasis. PLoS Neglected Tropical Diseases 5(6): e1151.

9. Kaiser M, Bray MA, Cal M, Bourdin Trunz B, Torreele E, Brun R (2011). Antitrypanosomal activity of fexinidazole, a new oral nitroimidazole drug candidate for treatment of sleeping sickness. Antimicrobial Agents and Chemotherapy 55(12): 5602-5608.

10. Matthews KR (2015). 25 years of African trypanosome research: From description to molecular dissection and new drug discovery. Molecular and Biochemical Parasitology 200(1-2): 30-40.

11. Steverding D, Sexton DW, Wang X, Gehrke SS, Wagner GK, Caffrey $C R$ (2012). Trypanosoma brucei: chemical evidence that cathepsin $L$ is essential for survival and a relevant drug target. International Journal for Parasitology 42(5): 481-488.

12. Martinez-Mayorga K, Byler KG, Ramirez-Hernandez Al, TerrazasAlvares DE (2015). Cruzain inhibitors: efforts made, current leads and a structural outlook of new hits. Drug Discovery Today 20(7): 890-898.

13. Alsford S, Eckert S, Baker N, Glover L, Sanchez-Flores A, Leung KF, Turner DJ, Field MC, Berriman M, Horn D (2012). High-throughput decoding of antitrypanosomal drug efficacy and resistance. Nature 482(7384): 232-236. 
14. Ch'ng JH, Liew K, Goh AS, Sidhartha E, Tan KS (2011). Drug-induced permeabilization of parasite's digestive vacuole is a key trigger of programmed cell death in Plasmodium falciparum. Cell Death and Disease 2:e216.

15. Koh H, Aye H, Tan K, He C (2015). The lysosomotropic drug LeuLeuOMe induces lysosome disruption and autophagy-independent cell death in Trypanosoma brucei. Microbial Cell 2(8): 288-298.

16. Thiele DL, Lipsky PE (1986). The immunosuppressive activity of Lleucyl-L-leucine methyl ester: selective ablation of cytotoxic lymphocytes and monocytes. Journal of Immunology 136(3): 1038-1048. doi.

17. Cirman T, Oresic K, Mazovec GD, Turk V, Reed JC, Myers RM, Salvesen GS, Turk B (2004). Selective disruption of lysosomes in HeLa cells triggers apoptosis mediated by cleavage of Bid by multiple papain-like lysosomal cathepsins. The Journal of Biological Chemistry 279(5): 3578-3587.

18. Taylor-Brown E, Hurd H (2013). The first suicides: a legacy inherited by parasitic protozoans from prokaryote ancestors. Parasites and Vectors 6:108.
19. Proto WR, Coombs GH, Mottram JC (2013). Cell death in parasitic protozoa: regulated or incidental? Nature Reviews Microbiology 11(1): 58-66.

20. Pays E, Vanhollebeke B, Uzureau P, Lecordier L, Perez-Morga D (2014). The molecular arms race between African trypanosomes and humans. Nature Reviews Microbiology 12(8): 575-584.

21. Perez-Morga D, Vanhollebeke $B$, Paturiaux-Hanocq F, Nolan DP, Lins L, Homble F, Vanhamme L, Tebabi P, Pays A, Poelvoorde P, Jacquet A, Brasseur R, Pays E (2005). Apolipoprotein L-I promotes trypanosome lysis by forming pores in lysosomal membranes. Science 309(5733): 469-472.

22. Horn D (2014). Antigenic variation in African trypanosomes. Molecular and Biochemical Parasitology 195(2): 123-129.

23. Engstler $M$, Pfohl T, Herminghaus S, Boshart M, Wiegertjes $G$, Heddergott N, Overath $P$ (2007). Hydrodynamic flow-mediated protein sorting on the cell surface of trypanosomes. Cell 131(3): 505-515.

24. Alsford S, Field MC, Horn D (2013) Receptor mediated endocytosis for drug delivery in African Trypanosomes: fulfilling Paul Ehrlich's vision of chemotherapy. Trends in Parasitology 29(5): 207-212. 